Abanico Veterinario. Enero-Diciembre 2021; 11:1-16. http://dx.doi.org/10.21929/abavet2021.23

Artículo Revisión. Recibido: 19/11/2020. Aceptado: 27/04/2021. Publicado: 15/05/2021. Clave: e2020-91.

\title{
Frecuencia de leptospirosis en equinos: revisión de literatura
}

\section{Frequency of Leptospira spp serovars reported in horses: a literature review}

\section{Toriz-Suarez Oscar ${ }^{1} \mathrm{ID}$, Pérez-Rivero Juan*2 ID, Herrera-Barragán Antonio ${ }^{2 \mathrm{ID}}$, Torres-Barranca Jorge 2 ID, Lombardero-Goldaracena German 2 ID}

\begin{abstract}
${ }^{1}$ Maestría en Ciencias Agropecuarias Universidad Autónoma Metropolitana Unidad Xochimilco; 2Departamento de Producción Agrícola y Animal. Universidad Autónoma Metropolitana Unidad Xochimilco. Calzada del hueso 1100, Coyoacán CDMX, CP. 04960. *Autor para correspondencia: Juan José PérezRivero; Departamento de Producción Agrícola y Animal. Edificio Wbis, piso 3. Universidad Autónoma Metropolitana-Xochimilco Calzada del hueso 1100, Coyoacán CDMX, CP. 04960. Correo electrónico: jjperez1_1999@yahoo.com,_miguel.tosu@gmail.com, jitorres@correo.xoc.uam.mx , jlombardero@correo.xoc.uam.mx

jherrerab@correo.xoc.uam.mx,
\end{abstract}

\section{Resumen}

La leptospirosis equina ha sido asociada con abortos, insuficiencia renal y uveítis, lo cual constituye un problema de salud y económico, sin embargo, de manera general es asintomática, lo cual provoca que dicha enfermedad sea de poco interés en equinos en comparación con otras especies domésticas y silvestres. En la actualidad la situación epidemiológica real de leptospirosis en equinos se desconoce; al igual que la enfermedad se desarrolla en estos animales. Se realizó una búsqueda de literatura científica; con el objetivo de conocer cuáles son las serovariedades del género leptospira reportadas en equinos desde 2010 a 2020. En la revisión de literatura se seleccionaron 21 artículos, en los cuales se identificó que Leptospira bratislava, es la que se reportó con mayor frecuencia (11.43\%, IC95\%: 6-19.1\%), seguida por Leptospira icterohaemorrhagiae (9.52\%, IC95\%: 4.6-16.8\%).

Palabras clave: hospedero, Infección, uveítis, zoonosis.

\begin{abstract}
Equine leptospirosis has been associated with abortions, renal failure and uveitis, which constitutes a health and economic problem. However, it is generally asymptomatic, which makes this disease of little interest in equines in comparison with other domestic and wild species. At present, the real epidemiological situation of leptospirosis in equines is unknown; just as the disease develops in these animals. A search of scientific literature was carried out; with the objective of knowing which are the serovars of genus leptospira reported in equines from 2010 to 2020. In the literature review, 21 articles were selected, in which it was identified that Leptospira Bratislava, is the most frequently reported (11.43\%, IC95\%: 6-19.1\%) is in contact with equines, followed by Leptospira icterohaemorrhagiae (9.52\%, IC95\%: 4.6-16.8\%).
\end{abstract}

Keywords: host, Infection, uveitis, zoonosis. 


\section{INTRODUCCIÓN}

La leptospirosis descrita en 1886 por el médico alemán Adolph Weil, es en la actualidad una de las enfermedades infecciosas de tipo zoonótico de más amplia distribución a nivel mundial, es causada por bacterias del género Leptospira spp (Adler y de la Peña, 2010). La frecuencia de leptospirosis suele asociarse con mala higiene por lo que su presencia es más frecuente en regiones donde son comunes las actividades como la agricultura, la manufactura de productos de origen animal, la silvicultura, la ganadería, la minería, etcétera (Torres et al., 2016).

Esta enfermedad tiene relevancia para la salud pública y animal, debido a la participación de hospederos naturales y accidentales, los cuales son importantes para su mantenimiento. Los roedores son considerados los principales hospederos, además de los bovinos, equinos, cerdos, ovejas, cabras, perros y gatos; así como mamíferos silvestres (Andersen et al., 2016). Algunos de éstos cumplen la función de portadores, los cuales pueden ser capaces de eliminar a la bacteria mediante la orina como lo hacen los individuos enfermos, sin presentar signos clínicos detectables (Wood et al., 2018; Ellis, 2014; Moral et al., 2014).

En los equinos, la leptospirosis se asocia de manera común con enfermedades genitourinarias y oftálmicas en las cuales se pueden apreciar daños hacia la placenta y el feto, los riñones y los ojos. Por lo general, el cuadro clínico es de carácter leve o subclínico; sin embargo, en la forma aguda se presenta depresión, ictericia, pirexia, mientras que en infecciones graves se observan abortos, enfermedades neonatales o el nacimiento de un potro sano con anticuerpos contra esta bacteria. En algunos casos se presenta mortalidad perinatal, además de trastornos respiratorios y cuadros de uveítis recurrente (ERU) o iridociclitis (Divers, et al., 2019; Arent y Kedzierska-Mięszkowska, 2013; Verma et al., 2010).

Los equinos se infectan al consumir agua contaminada con leptospiras; de igual forma como cualquier especie al entrar en contacto con la orina $u$ otros fluidos de animales infectados. Estas bacterias tienen la capacidad de entrar al organismo a través de las mucosas, abrasiones en piel y por vía transplacentaria, para de manera posterior puedan alojarse principalmente en los túbulos renales de los animales, en donde se encuentran colonizando (Khalili et al., 2109).

De manera posterior, las bacterias son eliminadas por la orina, contaminan los ríos, lagos y demás fuentes hídricas que son utilizadas para actividades destinadas al comercio, agricultura, ganadería, incluido para el consumo del ser humano, de esta manera se facilita la infección en humanos y animales (Pulido et al., 2014). En ciertas ocasiones algunos productos, tejidos y fluidos de estos animales pueden actuar como una fuente de infección.

Hamond et al. (2013), propone otra vía no convencional de transmisión de la enfermedad, mediante el contagio sexual, debido a que encontró la presencia de ADN, de Leptospira spp en semen equino, en el $50 \%$ de muestras evaluadas y frecuencia de $60 \%$ para Leptospira bratislava y Leptospira copenhageni. Cabe mencionar que, aunque la 
exposición a leptospira es común, el desarrollo de la enfermedad en equinos se presenta en raras ocasiones (Malalana, 2019).

En la actualidad no se conoce con exactitud si las condiciones en el riñón del caballo alteran las funciones fisiológicas de la bacteria y, por lo tanto, afectan su supervivencia, generan reducción de su patogenia, o si existen diferencias en la infección entre machos y hembras (Hamond et al., 2012a).Sin embargo, mediante estudios histopatológicos en riñones de caballos jóvenes, se ha logrado observar la formación de petequias e infiltración linfocítica en los túbulos proximales y glomérulos. También se ha reportado que la serovariedad Pomona, al alojarse en dichos órganos ocasiona fiebre e insuficiencia renal aguda (Verma et al., 2010).

El objetivo de esta revisión de literatura fue conocer la frecuencia de la leptospirosis en equinos reportada en la literatura de los últimos 10 años.

\section{METODOLOGÍA}

Se realizó una revisión en buscadores web como Pubmed, Science direct, Scielo, de la literatura científica arbitrada y publicada en los últimos 10 años (2010-2020), se utilizaron las palabras clave: frecuencia, equinos, Leptospira spp, leptospirosis, infección, diagnóstico, uveítis y la combinación de éstas.

Se obtuvieron de los artículos consultados los reportes de serovariedad, país y continente; con base en lo anterior, se calculó la frecuencia general de los animales positivos y la frecuencia de las serovariedades.

Después se utilizó el programa Epilnfo $7 \AA$ para analizar los datos, conocer las frecuencias e intervalos de confianza al 95\% (IC 95\%) de la información obtenida de los artículos revisados (CDC, 2016).

\section{RESULTADOS}

En una muestra representativa de 21 artículos, en donde se detectó alguna serovariedad de Leptospira en 7218 caballos (Tabla 1). En estos se encontraron 40 serovariedades (Tabla 2) siendo la Leptospira Bratislava la que se reporta con mayor frecuencia con 11.43\% (IC95\%: 6-19.1\%), seguida por Leptospira icterohaemorrhagiae con 9.52\%, (IC95\%: 4.6-16.8\%); lo cual sugiere que estas serovariedades reportadas son las que más se encuentran en contacto con equinos.

De los 21 artículos analizados, se reportó que Brasil es el país donde más se han realizado estudios sobre la leptospirosis equina durante la última década, con $47.62 \%$ (IC95\%: 25.71-70.22\%) como se muestra en la Tabla 3.

En la Tabla 4, se muestran las serovariedades reportadas en la literatura consultada en equinos durante los últimos diez años. 
Tabla 1. Frecuencias de caballos reportados con leptospirosis

\begin{tabular}{|c|c|}
\hline Autor & Animales usados en el estudio(n) \\
\hline Ali, 2012 & 409 \\
\hline Alves, 2016 & 100 \\
\hline Arent y Kedzierska-Mięszkowska, 2013 & 620 \\
\hline Bedoya et al., 2013 & 293 \\
\hline De Oliveira et al., 2014 & 257 \\
\hline Hamond et al., 2012 & 119 \\
\hline Hamond et al., 2013 & 10 \\
\hline Hamond et al., 2015 & 206 \\
\hline Malalana et al., 2019 & No disponible \\
\hline Martins et al., 2017 & 54 \\
\hline Méndez et al., 2013 & 24 \\
\hline Peixoto Ribeiro et al., 2018 & 1640 \\
\hline Pinna et al., 2014 & 608 \\
\hline Pikalo et al., 2016 & 314 \\
\hline Rey Riaño et al., 2015 & 94 \\
\hline Siqueira et al., 2019 & 1200 \\
\hline Simbizi et al., 2016 & 663 \\
\hline Troncoso et al., 2013 & 55 \\
\hline Tsegay et al., 2016 & 418 \\
\hline Vera et al., 219 & 134 \\
\hline Total & 7218 \\
\hline
\end{tabular}

Tabla 2. Frecuencia de diferentes especies de leptospira reportadas en equinos en de 2010 a 2020

\begin{tabular}{cccccc}
\hline Leptospira & Frecuencia & $\%$ & \% Acumulado & \multicolumn{2}{c}{ Intervalo de confianza al 95\% } \\
\hline arborea & 1 & 0.95 & 0.95 & 0.02 & 5.19 \\
australis & 4 & 3.81 & 4.76 & 1.05 & 9.47 \\
autumnalis & 5 & 4.76 & 9.52 & 1.56 & 10.76 \\
ballum & 2 & 1.90 & 11.43 & 0.23 & 6.71 \\
bataviae & 1 & 0.95 & 12.38 & 0.02 & 5.19 \\
bataviae (sv. swart) & 1 & 0.95 & 13.33 & 0.02 & 5.19 \\
bratislava & 12 & 11.43 & 24.76 & 6.05 & 19.11 \\
bratislava jez-bratislava & 1 & 0.95 & 25.71 & 0.02 & 5.19 \\
butembo & 1 & 0.95 & 26.67 & 0.02 & 5.19 \\
canicola & 6 & 5.71 & 32.38 & 2.13 & 12.02 \\
celledoni & 1 & 0.95 & 33.33 & 0.02 & 5.19 \\
copenhageni & 6 & 5.71 & 39.05 & 2.13 & 12.02
\end{tabular}




\begin{tabular}{|c|c|c|c|c|c|}
\hline djasiman & 3 & 2.86 & 43.81 & 0.59 & 8.12 \\
\hline grippothyphosa (sv. duyster) & 1 & 0.95 & 44.76 & 0.02 & 5.19 \\
\hline grippotyphosa & 7 & 6.67 & 51.43 & 2.72 & 13.25 \\
\hline grippotyphosa moska V & 1 & 0.95 & 52.38 & 0.02 & 5.19 \\
\hline hardjo & 4 & 3.81 & 56.19 & 1.05 & 9.47 \\
\hline hardjo bovis & 1 & 0.95 & 57.14 & 0.02 & 5.19 \\
\hline hardjo prajitno & 3 & 2.86 & 60.00 & 0.59 & 8.12 \\
\hline hardjobovis/wolffi & 1 & 0.95 & 60.95 & 0.02 & 5.19 \\
\hline hardjoprajitno H89 & 1 & 0.95 & 61.90 & 0.02 & 5.19 \\
\hline hebdomadis & 2 & 1.90 & 63.81 & 0.23 & 6.71 \\
\hline icterohaemorrhagiae & 10 & 9.52 & 73.33 & 4.66 & 16.82 \\
\hline javanica & 1 & 0.95 & 74.29 & 0.02 & 5.19 \\
\hline panama & 2 & 1.90 & 76.19 & 0.23 & 6.71 \\
\hline patoc & 1 & 0.95 & 77.14 & 0.02 & 5.19 \\
\hline poi & 1 & 0.95 & 78.10 & 0.02 & 5.19 \\
\hline pomona & 9 & 8.57 & 86.67 & 3.99 & 15.65 \\
\hline portland-vere sinaloa & 1 & 0.95 & 87.62 & 0.02 & 5.19 \\
\hline pyrogenes & 1 & 0.95 & 88.57 & 0.02 & 5.19 \\
\hline sentot & 1 & 0.95 & 89.52 & 0.02 & 5.19 \\
\hline seramanga & 1 & 0.95 & 90.48 & 0.02 & 5.19 \\
\hline serjoe & 2 & 1.90 & 92.38 & 0.23 & 6.71 \\
\hline tarassovi & 3 & 2.86 & 95.24 & 0.59 & 8.12 \\
\hline tarassovi perepelitsin & 1 & 0.95 & 96.19 & 0.02 & 5.19 \\
\hline topaz & 1 & 0.95 & 97.14 & $0.02 \%$ & 5.19 \\
\hline wolffi & 2 & 1.90 & 99.05 & $0.23 \%$ & 6.71 \\
\hline zanoni & 1 & 0.95 & 100 & $0.02 \%$ & 5.19 \\
\hline
\end{tabular}

Tabla 3. Frecuencia de reportes por país con leptospirosis equina durante el periodo 2010 -2020

\begin{tabular}{|c|c|c|c|c|c|}
\hline \multirow{2}{*}{$\begin{array}{l}\text { País /continente } \\
\text { Alemania/Europa }\end{array}$} & \multirow{2}{*}{ 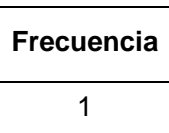 } & \multirow{2}{*}{$\begin{array}{c}\% \\
4.76\end{array}$} & \multirow{2}{*}{$\begin{array}{c}\text { \% Acumulado } \\
4.76\end{array}$} & \multicolumn{2}{|c|}{$\begin{array}{c}\text { Intervalo de confianza } \\
\text { al } 95 \%\end{array}$} \\
\hline & & & & 0.12 & 23.82 \\
\hline Brasil/América & 10 & 47.62 & 52.38 & 25.71 & 70.22 \\
\hline Chile/América & 1 & 4.76 & 57.14 & 0.12 & 23.82 \\
\hline Colombia/América & 2 & 9.52 & 66.67 & 1.17 & 30.38 \\
\hline Irán/Asia & 1 & 4.76 & 71.43 & 0.12 & 23.82 \\
\hline Italia/Europa & 1 & 4.76 & 76.19 & 0.12 & 23.82 \\
\hline México/América & 1 & 4.76 & 80.95 & 0.12 & 23.82 \\
\hline $\begin{array}{c}\text { Noroeste de Inglaterra y norte de } \\
\text { Gales/Europa }\end{array}$ & 1 & 4.76 & 85.71 & 0.12 & 23.82 \\
\hline Polonia/Europa & 1 & 4.76 & 90.48 & 0.12 & 23.82 \\
\hline Sudáfrica/África & 1 & 4.76 & 95.24 & 0.12 & 23.82 \\
\hline Sur de Etiopia/África & 1 & 4.76 & 100.00 & 0.12 & 23.82 \\
\hline Total & 21 & 100 & 100 & & \\
\hline
\end{tabular}


ABANICO VETERINARIO ISSN 2448-6132 abanicoacademico.mx/revistasabanico/index.php/abanico-veterinario Creative Commons (CC BY-NC 4.0) abanicoveterinario@gmail.com

Tabla 4. Frecuencia por país de infecciones por leptospira en equinos de 2010 a 2020

\begin{tabular}{|c|c|c|c|c|}
\hline Autor & País /continente & $\begin{array}{c}\text { Serovariedad encontrada } \\
\text { Leptospira: }\end{array}$ & $\begin{array}{c}\text { Frecuencia } \\
\text { reportada } \\
(\%)\end{array}$ & $\begin{array}{c}\% \text { animales positivos } \\
\text { Intervalo de confianza } \\
\text { al } 95 \%\end{array}$ \\
\hline \multirow{7}{*}{ Ali, 2012} & \multirow{7}{*}{ Irán/Asia } & pomona & 38.9 & \multirow{7}{*}{$\begin{array}{c}39.18 \\
35-43.6\end{array}$} \\
\hline & & grippotyphosa & 32.7 & \\
\hline & & & & \\
\hline & & icterohaemorrhagiae & 15.1 & \\
\hline & & canicola & 10.4 & \\
\hline & & hardjo & 1.7 & \\
\hline & & ballum & 1.04 & \\
\hline \multirow{3}{*}{ Alves et al., 2016} & \multirow{3}{*}{ Brasil/América } & patoc & 35.7 & \multirow{3}{*}{$\begin{array}{c}28.0 \\
20.1-37.5\end{array}$} \\
\hline & & butembo & 32.1 & \\
\hline & & sentot & 14.3 & \\
\hline \multirow{16}{*}{$\begin{array}{l}\text { Arent y Kedzierska- } \\
\text { Mięszkowska, } 2013\end{array}$} & \multirow{16}{*}{ Polonia/Europa } & grippotyphosa & 11.7 & \multirow{16}{*}{$\begin{array}{c}39.0 \\
35.3-43.0\end{array}$} \\
\hline & & serjoe & 4.5 & \\
\hline & & bratislava & 4.0 & \\
\hline & & poi & 3.7 & \\
\hline & & pomona & 3.6 & \\
\hline & & icterohaemorrhagiae & 2.9 & \\
\hline & & celledoni & 0.9 & \\
\hline & & cynopteri & 0.9 & \\
\hline & & ballum & 0.6 & \\
\hline & & hebdomadis & 0.6 & \\
\hline & & bataviae & 0.5 & \\
\hline & & hardjo & 0.4 & \\
\hline & & zanoni & 0.4 & \\
\hline & & autumnalis & 0.4 & \\
\hline & & canicola & 0.3 & \\
\hline & & australis & 0.3 & \\
\hline \multirow{6}{*}{ Bedoya et al., 2013} & \multirow{6}{*}{ Colombia/América } & bratislava & 53.3 & \multirow{6}{*}{$\begin{array}{c}66.70 \\
51.5-79.0\end{array}$} \\
\hline & & hardjo bovis & 28.5 & \\
\hline & & icterohaemorrhagiae & 26.3 & \\
\hline & & hardjo prajitno & 5.1 & \\
\hline & & grippotyphosa & 2.8 & \\
\hline & & pomona & 2.6 & \\
\hline Hamond et al., 2012b & Brasil/América & copenhageni & 43.7 & 71.4 \\
\hline
\end{tabular}


ABANICO VETERINARIO ISSN 2448-6132 abanicoacademico.mx/revistasabanico/index.php/abanico-veterinario Creative Commons (CC BY-NC 4.0) abanicoveterinario@gmail.com

\begin{tabular}{|c|c|c|c|c|}
\hline & & icterohaemorrhagiae & 27.8 & $62.7-78.8$ \\
\hline \multirow{2}{*}{ Hamond et al., 2013} & \multirow{2}{*}{ Brasil/América } & bratislava & 30.0 & 60 \\
\hline & & copenhageni & 30.0 & $31.3-83.2$ \\
\hline \multirow{2}{*}{ Hamond et al., 2014a } & \multirow{2}{*}{ Brasil/América } & australis & 54.4 & 47.8 \\
\hline & & icterohaemorrhagiae & 43.6 & $39.5-56.1$ \\
\hline \multirow{2}{*}{ Hamond et al., 2015} & \multirow{2}{*}{ Brasil/América } & australis & 46.4 & 44.7 \\
\hline & & pomona & 70.5 & $38.0-51.5$ \\
\hline \multirow{3}{*}{ Malalana et al., 2019} & \multirow{3}{*}{$\begin{array}{c}\text { Noroeste de Inglaterra } \\
\text { y norte de } \\
\text { Gales/Europa }\end{array}$} & bratislava & 19.4 & \multirow{3}{*}{$\begin{array}{c}51.4 \\
40.0-62.6\end{array}$} \\
\hline & & copenhageni y Australis & 6.9 & \\
\hline & & autumnalis & 8.3 & \\
\hline \multirow{2}{*}{ Martins et al., 2017} & \multirow{2}{*}{ Brasil/América } & australis & 69.2 & \multirow{2}{*}{$\begin{array}{c}48.1 \\
35.4-61.1\end{array}$} \\
\hline & & icterohaemorrhagiae & 30.8 & \\
\hline \multirow{9}{*}{ Méndez et al., 2013} & \multirow{9}{*}{ México/América } & hardjoprajitno H89 & 12.0 & \multirow{9}{*}{$\begin{array}{c}71 \\
50.8-85.0\end{array}$} \\
\hline & & wolffi & 12.0 & \\
\hline & & tarassovi perepelitsin & 41.0 & \\
\hline & & grippotyphosa moska $V$ & 4.0 & \\
\hline & & bratislava jez-bratislava & 8.0 & \\
\hline & & portland-vere sinaloa & 8.0 & \\
\hline & & hardjo prajitno & 29.0 & \\
\hline & & icterohaemorrhagiae & 12.0 & \\
\hline & & pomona & 42.0 & \\
\hline \multirow{6}{*}{$\begin{array}{l}\text { Peixoto Ribeiro et al., } \\
\qquad 2018\end{array}$} & \multirow{6}{*}{ Brasil/América } & serjoe & 5.9 & \multirow{6}{*}{$\begin{array}{c}32.7 \\
30.5-35.0\end{array}$} \\
\hline & & seramanga & 5.3 & \\
\hline & & djasiman & 3.9 & \\
\hline & & grippotyphosa & 3.9 & \\
\hline & & icterohaemorrhagiae & 3.6 & \\
\hline & & autumnalis & 2.6 & \\
\hline \multirow{2}{*}{ Pinna et al., 2014} & \multirow{2}{*}{ Brasil/América } & bratislava & 62.3 & \multirow{2}{*}{$\begin{array}{c}44.9 \\
41.0-48.9\end{array}$} \\
\hline & & copenhageni & 37.7 & \\
\hline \multirow{3}{*}{ Pikalo et al., 2016} & \multirow{3}{*}{ Alemania/Europa } & icterohaemorrhagiae & 11.1 & \multirow{3}{*}{$\begin{array}{c}17.20 \\
13.4-21.8\end{array}$} \\
\hline & & bratislava & 9.6 & \\
\hline & & grippotyphosa & 1.9 & \\
\hline \multirow{7}{*}{ Rey et al., 2015} & \multirow{7}{*}{ Colombia/América } & pomona & 41.5 & \multirow{7}{*}{$\begin{array}{c}76.6 \\
67.1-84.0\end{array}$} \\
\hline & & grippotyphosa & 24.5 & \\
\hline & & canicola & 16.0 & \\
\hline & & javanica & 23.4 & \\
\hline & & hardjo prajitno & 10.6 & \\
\hline & & tarassovi & 7.4 & \\
\hline & & hebdomadis & 7.4 & \\
\hline
\end{tabular}




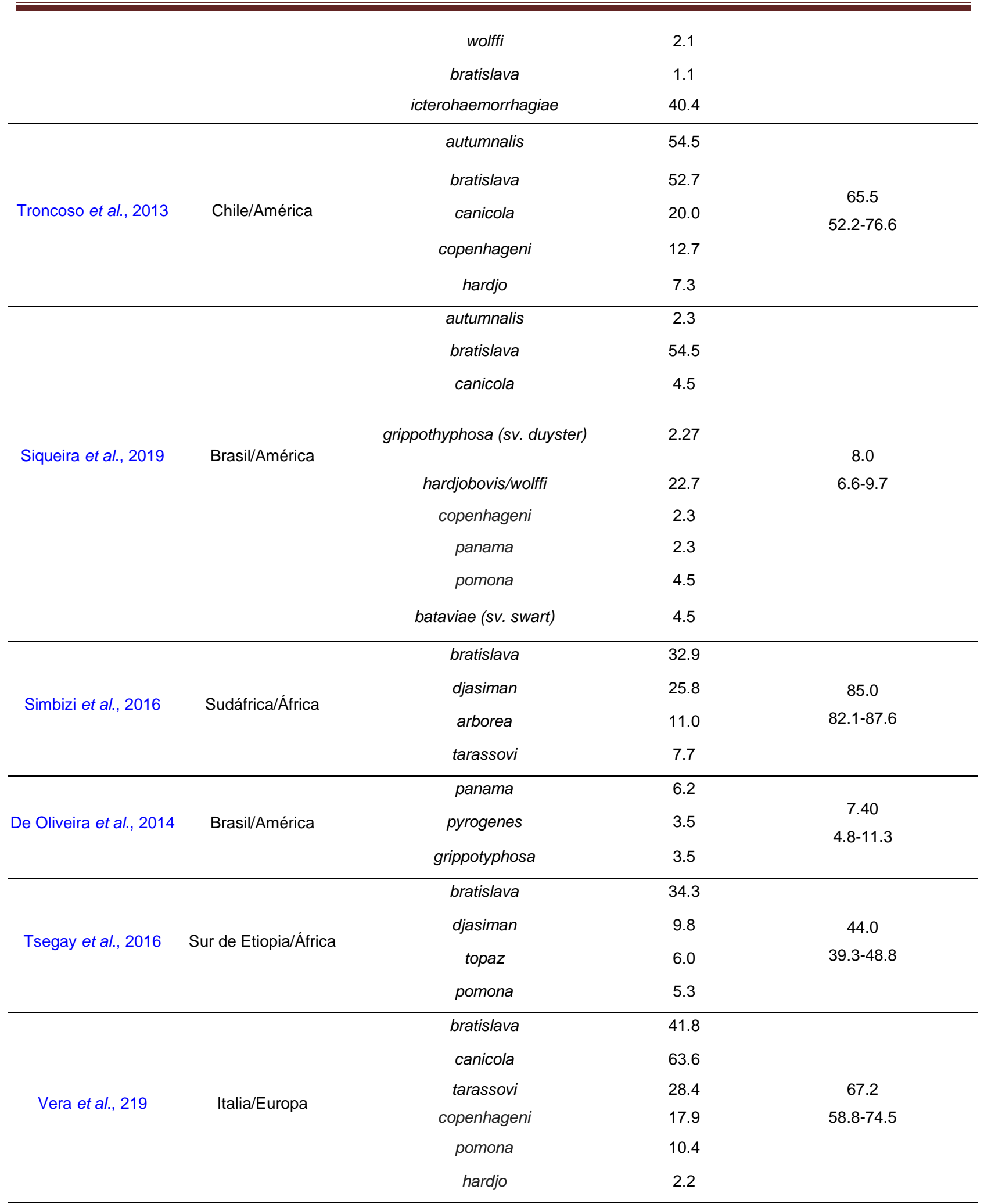




\section{DISCUSIÓN}

El conocer el verdadero valor de la frecuencia de Leptospira spp a partir de los artículos revisados es muy complicado, de ahí se calculó un intervalo de confianza para poder ubicar con una probabilidad del 95\%, un rango de valores entre los cuales se localizará el verdadero valor de la frecuencia de este agente en la población equina (Molina, 2013). En este estudio se identificaron 40 serovariedades de Leptospira spp en caballos. A pesar de que la Leptospira bratislava es la que más se ha reportado en la literatura de equinos en los últimos 10 años (Pinna et al., 2014), la Leptospira pomona es la que se considera como el agente específico para los équidos, puesto que cuando el individuo desarrolla la enfermedad, se pueden encontrar títulos elevados de anticuerpos contra esta última (Divers et al., 2019).

En los estudios serológicos en equinos se ha identificado que existe variación en la frecuencia de infección por Leptospira (Hamond et al., 2014b); las cuales pueden presentarse en los 4 continentes reportados en la muestra del estudio sin embargo, las infecciones que más se reportaron en la literatura en equinos de los últimos 10 años fueron causadas por Leptospira icterohaemorrhagiae, Leptospira pomona, Leptospira bratislava, Leptospira copenhageni y Leptospira grippotyphosa. Estos datos se obtuvieron de acuerdo con lo reportado en los artículos revisados, derivado de estudios, tanto clínicos, serológicos y de ecología de la enfermedad, lo cual ha permitido conocer la interfaz entre animal-ambiente-humano y su importancia para el concepto de una sola salud (Jaeger et al., 2019). Por otra parte, Bertelloni et al. (2019) mencionan que las características ambientales, como los humedales, estanques y canales presentes en el centro de Italia, asociadas con la presencia de animales domésticos y silvestres (estos últimos como reservorios de leptospiras) favorecen la diseminación de esta enfermedad, y se reportan frecuencias en cerdos de $19.74 \%$ y en bovinos de $13.03 \%$. Se conoce que las leptospiras son transmitidas de manera eficiente a través del agua, pudiendo sobrevivir por periodos largos en ambientes húmedos (Lourerio et al., 2013). Por su parte, Verma et al., 2019 demostraron la existencia de la interacción entre el patógeno y los animales domésticos y silvestres, mediante los asentamientos de agua, así como la presencia de esta bacteria en los riñones de los animales silvestres. Se ha sugerido que la adaptación de la serovariedad al hospedero, así como, la cercana convivencia con el ganado con los caballos puede influir en la frecuencia de exposición (Lowe, 2010). Como lo demuestran Pinna et al. (2014) Leptospira bratislava está adaptada a los equinos, ya que esta se ha asociado con problemas reproductivos en yeguas. Sin embargo, Leptospira bratislava y Leptospira muenchen, se han asociado con casos de infecciones en cerdos, caballos, bovinos y perros, y logrado identificar cepas similares en animales silvestres (Arent et al., 2016).

Witkswski et al. (2016) mencionan que las Leptospira grippotyphosa y Leptospira pomona se asocian con casos de uveítis recurrentes en equinos. Hashimoto et al. (2007) 
evaluaron la prevalencia de leptospirosis en equinos sin sinología clínica aparente, y utilizaron la pérdida de peso y depresión como criterios de inclusión, con lo cual obtuvieron $66.88 \%$ de frecuencia general; de este valor, el $23.36 \%$ se representó por anticuerpos contra Leptospira icterohaemorrhagiae y el $13.14 \%$ por Leptospira grippotyphosa. Mientras que Hamond et al. (2012b) encontraron 89.5 de seroprevalencia en caballos asintomáticos.

La gravedad de la enfermedad varía con la serovariedad y el animal afectado, cuando se presenta la enfermedad de manera subclínica, por lo general no se hace aparente el deterioro del animal; sin embargo, cuando la presencia de la enfermedad es de tipo crónico, son evidentes el deterioro físico y productivo de los animales (Ellis, 2015).

Leptospira pomona kennewicki es considerada como la responsable de la mayoría de las presentaciones clínicas en caballos norteamericanos, se encuentra asociada con enfermedades de la placenta y el feto, los riñones y los ojos (Divers et al., 2019); sin embargo, la presentación clínica puede resultar en aborto o en un potro enfermo. Esto se puede deber a la etapa gestacional de la yegua y al momento en el que ésta se infectó con la bacteria, así como su estado inmunológico (Bernard, 1993). Desde el punto de vista clínico, un estudio realizado en Suecia demostró que no hay asociación significativa entre la presencia de signos clínicos de enfermedad y títulos positivos a Leptospira bratislava o Leptospira Icterohaemorrhagiae con excepción con la asociación entre problemas respiratorios y fatiga con la Leptospira Bratislava (Båverud et al., 2009). Como es el caso de Rocha (2004) en el cual mediante la obtención de muestras de riñón de caballo logró los aislamientos y tipificación por serología; en donde se determinó la presencia de cepas de Leptospira australis y Leptospira pomona, estas dos cepas son las que más se han reportado en caballos portugueses.

Se ha asociado a la frecuencia de infección con Leptospira spp a las condiciones ambientales, incluidas las variaciones estacionales, como ejemplo se tiene que Leptospira icterohaemorrhagie, se presentan con mayor frecuencia entre los meses de octubre y diciembre, esto atribuido a que los roedores suelen invadir los establos y casas a finales del verano y otoño. Por otro lado el incremento de la frecuencia Leptospira bratislava se presenta entre abril y junio y en octubre a diciembre (Båverud et al., 2009). La leptospirosis es una zoonosis descuidada con distribución mundial, afecta muchas especies de mamíferos, incluyendo el ganado, provocando signos clínicos de carácter agudo en animales de todas las edades y etapas productivas, lo cual afecta de manera importante la producción animal. La incidencia anual de esta zoonosis en humanos no está bien determinada debido a la subnotificación, se estima como ejemplo en Uruguay que es de 15 por cada 100,000 habitantes. La enfermedad humana parece estar asociada el contacto con animales infectados, así como con las lluvias y las inundaciones en regiones endémicas (Zarantonelli et al., 2018). 


\section{CONCLUSIONES}

De manera general la literatura consultada permitió conocer la frecuencia de la enfermedad y las serovariedades encontradas en los caballos. La exposición a las diferentes serovariedades puede estar relacionada con la región en la que se encuentren los equinos, así como la presencia de uno o varios hospedadores específicos para estas serovariedades los cuales actúan como reservorio para que se presente la infección. Los caballos se infectan cuando consumen agua contaminada con alguna especie de Leptospira. Si bien la exposición a estos agentes es común en los caballos, la enfermedad sistémica es rara, los equinos pueden cursar de manera asintomática o presentar diferentes signos clínicos. Las especies de leptospiras reportadas de manera frecuente que pueden infectar a los caballos son, Leptospira bratislava, (11.43\%, IC95\%: 6-19.1\%) y con la Leptospira icterohaemorrhagiae (9.52\%, IC95\%: 4.6-16.8\%). Es necesario considerar en futuros estudios la investigación del impacto de la leptospirosis animal en la salud pública, animal y ambiental, para poder implementar programas de prevención y control geográficamente específicos de esta enfermedad.

\section{Declaración de conflictos de interés}

No existe ningún tipo de conflicto de interés

\section{Agradecimientos}

Al Consejo Nacional de Ciencia y Tecnología (CONACYT) de México por la beca otorgada a OTS para realizar estudios de Maestría en Ciencias Agropecuarias en la Universidad Autónoma Metropolitana Unidad Xochimilco.

\section{LITERATURA CITADA}

ADLER B, De la Peña Moctezuma A. 2010. Leptospira and leptospirosis. Veterinary Microbiology. 140(3-4):287-296. http://doi.org/10.1016/j.vetmic.2009.03.012

ALVES JRA, Oliveira KDS, Costa DF, Fernandez LG, Higino SSS, Alves CJ, Santos CSA, Batista AS. 2016. Epidemiological characterization of leptospirosis in horses in the state of Pernambuco, northeastern Brazil. Arquivos do Instituto Biológico. 86:1-5. http://doi.org/10.1590/1808- 2141657001032014

ALI H, Saeid S. 2012. Seroprevalence of leptospiral infection in horses, donkeys and mules in east Azerbaijan province. African Journal of Microbiology Research. 6(20):43844387. http://doi.org/10.5897/AJMRx11.034 
ANDERSEN, REU, Pipper PMC. 2016. Jensen, Global patterns of leptospira prevalence in vertebrate reservoir hosts. Journal of Wildlife Diseases. 52(3):468-477. http://doi.org/10.7589/2014-10-245

ARENT Z, Kedzierska-Mięszkowska S. 2013. Seroprevalence study of leptospirosis in horses in northern Poland. VetRecord. 172:269. https://doi.org/10.1136/vr.101239

ARENT Z, Frizzell C, Gilmore C, Allen A, Ellis WA. 2016. Leptospira interrogans serovars Bratislava and Muenchen animal infections: Implications for epidemiology and control. Veterinary Microbiology. 190: 19-26.

https://doi.org/10.1016/j.vetmic.2016.05.004

BÅVERUD V, Gunnarsson A, Engvall EO, Franzén P, Egenvall A. 2009. Leptospira seroprevalence and associations between seropositivity, clinical disease and host factors in horses. Acta Veterinaria Scandinavica. 51(1):15. http://doi.org/10.1186/1751-0147-5115

BEDOYA RMA, Jaimes SJ, Molina SL. 2013. Prevalencia de lepstospira spp. en equinos de la vereda Guatiguara del municipio de Piedecuesta Santander. REDVET. 14(11B): 16. https://www.redalyc.org/pdf/636/63632393019.pdf

BERNARD WV. 1993. Leptospirosis. The Veterinary clinics of North America. Equine practice. 9(2):435-444. https://doi.org/10.1016/s0749-0739(17)30410-8

BERTELLONI F, Cilia G, Turchi B, Pinzauti P, Cerri D, Fratini F. 2019. Epidemiology of leptospirosis in North-Central Italy: Fifteen years of serological data (2002-2016). Comparative Immunology, Microbiology and Infectious Diseases. 65:1422. https://doi.org/10.1016/j.cimid.2019.04.001

CDC. 2016.Centers for Disease Control and Prevention Epilnfo7 User Guide. https://www.cdc.gov/epiinfo/pdfs/UserGuide/El7Full.pdf

DE OLIVEIRA FILHO RB, Malta KC, Oliveira JMB, Assis Santana VL, Harrop MHV, Stipp DT, Pinheiro JW. 2014. Epidemiological Analysis of Leptospira spp. Infection in Equids from the Brejo Paraibano Microregion of Brazil. Journal of Equine Veterinary Science. 34(3):407-414. https://doi.org/10.1016/j.jevs.2013.08.001

DIVERS TJ, Chang YF, Irby NL, Smith JL, Carter CN. 2019. Leptospirosis: An important infectious disease in North American horses. Equine Veterinary Journal. 51(3):287292. https://doi.org/10.1111/evj.13069

ELLIS WA. 2014. Animal Leptospirosis. Leptospira and Leptospirosis. 99-137. https://doi.org/10.1007/978-3-662-45059-8_6 
ELLIS WA. 2015. Animal leptospirosis. Curr Top Microbiol Immunol. 387:99-137. https://doi.org/10.1007/978-3-662-45059-8_6

HASHIMOTO VY, Gonçalves DD, da Silva FG, de Oliveira RC, Alves LA, Reichmann P, de Freitas JC. 2007. Occurrence of antibodies against Leptospira spp. in horses of the urban area of Londrina, Paraná. Revista do Instituto de Medicina Tropical de São Paulo. 49(5):327-330. https://doi.org/10.1590/S0036-46652007000500010

HAMOND C, Martins G, Lilenbaum W, Madeiros MA. 2012a . PCR detection of leptospiral carriers among seronegative horses. VetRecord. 171:105-6. https://doi.org/10.1136/vr.e5022

HAMOND C, Martins G, Lilenbaum W. 2012 ${ }^{\text {b }}$ Subclinical leptospirosis may impair athletic performance in racing horses. Tropical Animal Health and Production. 44(8):19271930. https://doi.org/10.1007/s11250-012-0158-5

HAMOND C, Martins G, Medeiros MA, Lilenbaum W. 2013. Presence of leptospiral DNA in semen suggests venereal transmission in Horses. Journal of Equine Veterinary Science. 33(12):1157-1159. https://doi.org/10.1016/j.jevs.2013.03.185

HAMOND C, Martins G, Bremont S, Medeiros MA, Bourhy $\mathrm{P}$, Lilenbaum W. $2014^{a}$. Predominance of Leptospira interrogans serovar Bratislava DNA in vaginal fluid of mares suggests sexual transmission of leptospirosis. Animal Reproduction Science. 151(3-4):275-279. https://doi.org/10.1016/j.anireprosci.2014.10.019

HAMOND C, Pinna A, Martins G, Lilenbaum W. 2014b. The role of leptospirosis in reproductive disorders in horses. Tropical animal health and production. 46(1):1-10. https://doi.org/10.1007/s11250-013-0459-3

HAMOND C, Pestana CP, Rocha-de-Souza CM, Cunha LER, Brandão FZ, Medeiros MA, Lilenbaum W. 2015. Presence of leptospires on genital tract of mares with reproductive problems. Veterinary Microbiology. 179(3-4):264269. https://doi.org/10.1016/j.vetmic.2015.06.014

JAEGER L, Pestana C, Correia L, Carvalho-Costa F, Medeiros M, Lilenbaum W. 2019. Novel MLST Sequence Types of pathogenic Leptospira spp.: opening the black box of animal leptospirosis in Brazil. Acta Tropica. https://doi.org/10.1016/j.actatropica.2019.05.025

KHALILI M, Sakhaee E, Amiri FB, Safat AA, Afshar D, Esmaeili S. 2019. Serological evidence of leptospirosis in Iran; A systematic review and meta-analysis. Microbial Pathogenesis. https://doi.org/10.1016/j.micpath.2019.103833 
LOUREIRO A P, Hamond C, Lilenbaum W. 2013. Leptospirosis in horses. Veterinary Record. 172(18):479-480. https://doi.org/10.1136/vr.f2824

LOWE RC. 2010. Equine uveitis: a UK perspective. Equine veterinary journal. Supplement. (37):46-49. https://doi.org/10.1111/j.2042-3306.2010.tb05634.x

MALALANA F. 2019. Leptospirosis in horses: A European perspective. Equine Veterinary Journal. 51(3):285-286. https://doi.org/10.1111/evj.13022

MARTINS G, Loureiro AP, Libonati H, Lilenbaum W. 2017. Humoral response in naturally exposed horses after leptospiral vaccination. Journal of Equine Veterinary Science. 57:24-28. https://doi.org/10.1016/j.jevs.2017.06.005

MÉNDEZ C, Benavides A, Esquivel A, Aldama A, Torres J, Gavaldon D, Meléndez P, Moles LP. 2013. Pesquisa serológica de Leptospira en roedores silvestres, bovinos, equinos y caninos en el noreste de México. Revista de Salud Animal. 35(1):25-32. http://scielo.sld.cu/scielo.php?script=sci_arttext\&pid=S0253-570X2013000100004

MOLINA AM. 2013. El significado de los intervalos de confianza. Rev Pediatr Aten Primaria; 15(57):91-94. https://www.redalyc.org/pdf/3666/366638747016.pdf

MORAL M, Laplume H, Sardi F, Samartino L, Vanasco B, San Juan J, Casas N. 2014. Enfermedades infecciosas leptospirosis. Ministerio de Salud de la Nación. http://www.msal.gob.ar/images/stories/bes/graficos/0000000489cnt-guia-medicaleptospirosis.pdf consultado el 14/08/2018

PEIXOTO RIBEIRO TM, Correia L, Hofstaetter Spohr KA, Aguiar DM, Martins G, de Sá Jayme V. 2018. Risk factors associated with seroreactivity against Leptospira spp. in horses from Brazilian Amazon. Journal of Equine Veterinary Science. 68:59-62. . https://doi.org/10.1016/j.jevs.2018.05.197

PIKALO J, Sattler T, Eichinger M, Loitsch A, Sun H, Schmoll F, Schusser GF. 2016. Vorkommen von antikörpern gegen leptospiren bei pferden im mitteldeutschen raum [Occurrance of antibodies against Leptospira in horses in Middle Germany]. Berliner und Munchener tierarztliche Wochenschrift. 129(5-6):202-208. https://www.vetline.de/system/files/frei/BMW_2016_05_06_0202_onl300.pdf

PULIDO VA, Carreño BG, Mercado RM, Ramírez BP. 2014. Situación epidemiológica de la leptospirosis humana en centro américa, sur américa y el caribe. Universitas Scientiarum. 19(3):246-264. https://wwww.redalyc.org/articulo.oa?id=499/49931030006 
PINNA A, Martins G, Hamond C, Medeiros MA, de Souza GN, Lilenbaum W. 2014. Potential differences between Leptospira serovars, host-adapted (Bratislava) and incidental (Copenhageni), in determining reproductive disorders in embryo transfer recipient mares in Brazil. The Veterinary Record. 174(21):531. https://doi.org/10.1136/vr.101444

REY RIAÑO LA, Pineda RNF, Góngora OA, Parra AJL, Patiño BRE. 2015. Nota serológica a Leptospira spp. En equinos aparentemente sanos en municipios del Meta y Guaviare, Colombia. Revista Lasallista de Investigación. 12(1):154-161. https://www.redalyc.org/articulo.oa?id=695/69542290015

ROCHA T. 2004. Microbiological and serological study of leptospirosis in horses at slaughter: first isolations. Research in Veterinary Science. 76(3):199202. https://doi.org/10.1016/j.rvsc.2003.12.003

SIMBIZI V, Saulez MN, Potts A, Lötter C, Gummow B. 2016. A study of leptospirosis in South African horses and associated risk factors. Preventive Veterinary Medicine. 134: 6-15. https://doi.org/10.1016/j.prevetmed.2016.09.019

SIQUEIRA CC, Fraga DBM, Chagas-Junior AD, Athanazio DA, Silva MMN, Cerqueira RB, Ayres MCC. 2019. Seroprevalence and risk factors associated with equine leptospirosis in the metropolitan region of Salvador and Recôncavo Baiano region, Bahia state (NE Brazil). Tropical Animal Health and Production. 52:31-39. https://doi.org/10.1007/s11250019-01956-5

TORRES CM, Hernández BS, Agudelo FP, Arroyave SE, Zaval CJ. Puerto FI. 2016. Revisión actual de la epidemiologia de la leptospirosis. Revista Médica del Instituto Mexicano del Seguro Social. 54(5):620-5. https://www.medigraphic.com/pdfs/imss/im2016/im165k.pdf

TRONCOSO TI, Toro BJ, Guzmán CA, Fuentealba OJ, Fischer WC. 2013. Evaluación serológica de Leptospira interrogans en equinos pertenecientes a un centro ecuestre de la provincia de Linares, Chile. Revista CES Medicina Veterinaria y Zootecnia. 8(2):101107. http://www.scielo.org.co/pdf/cmvz/v8n2/v8n2a10.pdf

TSEGAY K, Potts AD, Aklilu N, Lddototter C, Gummow B. 2016. Circulating serovars of Leptospira in cart horses of central and southern Ethiopia and associated risk factors. Preventive Veterinary Medicine. 125(1): 106-115.

http://dx.doi.org/10.1016/j.prevetmed.2016.01.009 
VERA E, Taddei S, Cavirani S, Schiavi J, Angelone M, Cabassi CS, Schiano E, Quintavalla F. 2019. Leptospira Seroprevalence in bardigiano horses in northern Italy. Animals: an open access journal from MDPI. 10(1):23.

https://doi.org/10.3390/ani10010023

VERMA A, Kumar P, Babb K, Timoney J F, Stevenson B. 2010. Cross-Reactivity of Antibodies against Leptospiral Recurrent Uveitis-Associated Proteins A and B (LruA and LruB) with Eye Proteins. PLOS Neglected Tropical Diseases. 4(8):778. https://doi.org/10.1371/journal.pntd.0000778

VERMA A, Beigel B, Smola CC, Kitts-Morgan S, Kish D, Nader P, Goss R. 2019. Evidence of Leptospiral Presence in the Cumberland Gap Region. PLOS Neglected Tropical Diseases. 13(12): e0007990. https://doi.org/10.1371/journal.pntd.0007990

WITKOWSKI L, Cywinska A, Paschalis-Trela K, Crisman M, Kita J. 2016. Multiple etiologies of equine recurrent uveitis - A natural model for human autoimmune uveitis: A brief review. Comparative Immunology, Microbiology and Infectious Diseases. 44:1420. https://doi.org/10.1016/j.cimid.2015.11.004

WOOD PL, Steinman M, Erol E, Carter C, Christmann U, Verma A. 2018. Lipidomic analysis of immune activation in equine leptospirosis and Leptospira-vaccinated horses. PLOS ONE. 13(2). https://doi.org/10.1371/journal.pone.0193424

ZARANTONELLI L, Suanes A, Meny P, Buroni F, Nieves C, Salaberry X, Briano C, Ashfield N, Silveira S, Dutra F, Easton C, Fraga M, Giannitti F, Hamond C, MaciasRioseco M, Menendez C, Mortola A, Picardeau M, Quintero J, Rios C, Rodríguez V, Romero A, Varela G, Rivero R, Schelotto F, Riet-Correa F, Buschiazzo A. 2018. Isolation of pathogenic Leptospira strains from naturally infected cattle in Uruguay reveals high serovar diversity, and uncovers a relevant risk for human leptospirosis. PLoS Negl Trop Dis. 12(9): e0006694. https://doi.org/10.1371/journal.pntd.0006694 